\title{
Multiple routes to pluripotency
}

The reprogramming of somatic cells to a pluripotent state, which is defined by the ability of a cell to differentiate into derivatives of all three embryonic germ layers, has great potential for the study and treatment of disease. Although reprogramming can be achieved through the forced expression of four transcription factors commonly known as OSKM (OCT4 (also known as POU5F1), SOX2, KLF4 and MYC) - a thorough and complete molecular characterization of the reprogramming process has been missing. Five papers now report extensive transcriptomic, epigenomic and proteomic analyses of reprogramming, revealing the existence of several reprogramming routes and showing that induced pluripotent stem (iPS) cells exist in multiple unique cell states.

In two studies published in Nature (Hussein et al. and Tonge et al.), Nagy and colleagues used a secondary reprogramming system, in which the starting differentiated cells are mouse iPS cell-derived fibroblasts containing doxycycline-inducible OSKM. This system produces enough reprogrammed cells to enable detailed molecular analyses to be carried out. Previous secondary reprogramming studies provided evidence that reprogramming occurs in multiple steps via transitions through defined transcriptional and chromatin states.

To characterize these steps, the authors generated multiple omic profiles at specific time points. Such profiles included the transcriptome (for both long and short transcripts), the global and cell-surface proteome, and the epigenome. The latter tracked different chromatin marks, specifically activating histone $\mathrm{H} 3$ lysine 4 trimethylation (H3K4me3), repressive H3K27me3 and $\mathrm{H} 3 \mathrm{~K} 36 \mathrm{me} 3$, which is indicative of transcriptional elongation.

Combined analyses of such profiles led to the characterization of two paths of reprogramming: one that leads to embryonic stem (ES) cell-like cells, and one that leads to an alternative stable pluripotent state. Cells in this alternative state were termed 'F-class cells', as they formed 'fuzzy' colonies in culture. The initial activation of OSKM led to a transient 'opening' of chromatin caused by a global loss of $\mathrm{H} 3 \mathrm{~K} 27 \mathrm{me}$, with levels reaching a nadir 8 days after induction. Global levels of H3K27me3 were then gradually restored during the transition to both ES cell-like and F-class states, albeit with different patterns.

If OSKM transgene levels were lowered after day 8 , by reducing the doxycycline concentration, reprogramming to the ES cell-like state was facilitated. This reprogramming route was characterized by a late phase (after day 8) of extensive DNA demethylation and gain of $\mathrm{H} 3 \mathrm{~K} 4 \mathrm{me} 3$ on genes, the activation of which stabilizes the ES cell-like state. If OSKM transgene levels remained high after day 8 , this led to F-class cells, which were characterized by the absence of global DNA demethylation and the activation of a different set of genes.

Notably, F-class cells stably expressed the pluripotency marker NANOG and were capable of forming teratomas that were indistinguishable from those derived from ES cells, but they did not contribute to chimaeras when injected into mouse embryos, confirming that they define a distinct pluripotent state.

Three related studies published in Nature Communications report detailed analyses of the genome-wide changes in DNA methylation (Lee et al.), the proteome (Benevento et al.) and small RNA expression (Clancy et al.) that occur during secondary reprogramming at several defined time points. Of note, microRNA changes occurred in two waves, early and late, which correlated with the changes in histone and DNA methylation seen in the other studies. The studies also report that F-class cells expressed a distinct subset of microRNAs and proteins, and lost both epithelial and ES cell-specific adhesion proteins.

These studies provide a comprehensive analysis of the progressive molecular changes that occur during reprogramming, and should enable researchers to manipulate this process more efficiently. Moreover, the possibility of obtaining different pluripotent states provides the potential to create artificial cells with unique properties that are suitable for specific applications.

Kim Baumann, Senior Editor, Nature Reviews Molecular Cell Biology This article is modified from the original in Nature Rev. Mol. Cell Biol. (http://dx.doi.org/10.1038/nrm3927).

\section{ORIGINAL RESEARCH PAPERS}

Hussein, S. M. I. et al. Genome-wide characterization of the routes to pluripotency. Nature 516, 198-206 (2014) | Tonge, P. D. et al. Divergent reprogramming routes lead to alternative stem-cell states. Nature 516, 192-197 (2014) | Lee, D.-S. et al. An epigenomic roadmap to induced pluripotency reveals methylation as a reprogramming modulator. Nature Commun. 5, 5619 (2014)| Benevento, M. et al. Proteome adaptation in cell reprogramming proceeds via distinct transcriptional networks. Nature Commun. 5, 5613 (2014) | Clancy, J. L. et al. Small RNA changes en route to distinct cellular states of induced pluripotency. Nature Commun. 5, 5522 (2014) 